\title{
Three-Dimensional Architecture of the Acetabular Transverse Ligament and its Connection with the Acetabular Labrum
}

\author{
N. Kaku ${ }^{1}$, T. Shimada ${ }^{2}$ R. Nogami ${ }^{3}$, H. Tagomori ${ }^{1}$, H. Tsumura ${ }^{1}$ \\ 1 Department of Orthopaedic Surgery, Faculty of Medicine, Oita University, Yufu City, Oita, Japan \\ 2 Oita College of Judo Therapy and Acupuncture-Moxibustion, Oita City, Japan \\ 3 Oita University Graduate School of Medicine, Graduate School of Orthopedic Surgery, Yufu City, Oita, Japan
}

\section{CORRESPONDING AUTHOR:}

Nobuhiro Kaku

Department of Orthopaedic Surgery

Faculty of Medicine, Oita University

Idaigaoka Hazama-machi

879-5593 Yufu City, Oita, Japan

E-mail: nobuhiro@oita-u.ac.jp

DOI:

10.32098/mltj.03.2020.01

LEVEL OF EVIDENCE: 5

\begin{abstract}
SUMMARY
Background. We clarified the microstructure of the acetabular transverse ligament in terms of the construction and arrangement of the collagen fibers constituting the ligament and their connection with the acetabular labrum using light and scanning electron microscopy (SEM).

Methods. Deparaffinized blocks were treated with $2 \mathrm{~N} \mathrm{NaOH}$ to digest the cell matrix, allowing the collagen fibers constituting the ligament and cartilage to be observed under SEM.

Results. The acetabular transverse ligament had parallel collagen fibers with a width of 30-50 $\mathrm{m}$, which consisted of bundles with type I collagen fibrils. The collagen fibers of the transverse ligament were interwoven between the cartilage tissues of the acetabular labrum, forming a layered structure. These components seemed to be directly joined to each other histologically. However, in SEM images, the collagen fibers were not directly connected; rather, fine collagen fibers with varying diameters extending from the structures were interwoven.

Conclusions. The acetabular transverse ligament had a distinctly different microstructure than the acetabular labrum. At the connection of both tissues, we found that the microstructure was not a direct adhesion but rather a structural bond, at least with respect to collagen. The results suggest that ligaments potentially attach to tissues other than bone.
\end{abstract}

\section{KEY WORDS}

Acetabular labrum; acetabular transverse ligament; collagen fibrils; microstructure; scanning electron microscopy.

\section{BACKGROUND}

The acetabular labrum of the hip joint is not continuous, but rather horseshoe-shaped, with its base connected by the acetabular transverse ligament. This is unlike the shoulder joint with the labrum, which exists all around the glenoid fossa. The acetabular transverse ligament crosses the acetabular incision and visibly connects to the acetabular labrum at the anterior and posterior parts of the acetabular notch (1). The ligament adheres not only to the acetabular labrum but also to the bone, forming a sucker-like structure surrounding the lower part of the femoral head. It has elements that function in conjunction with the acetabular labrum which helps prevent the microinstability of the hip joint $(2,3)$. The shape of the acetabulum varies among ethnic groups(4), and the acetabular transverse ligament and labrum are easily affected, such as in cases of inversion or damage to them, when acetabular dysplasia is present $(5,6)$.

The acetabular diameter is smaller than the femoral head diameter when unloaded (7). The anterior and posterior corners of the lunar surface open with loading to disperse the load, but the acetabular transverse ligament is positioned anteriorly and posteriorly and is thought to spread the force between the corners and participate in load distribution (1). However, a study reported that the amount of 
load on the lunar surface does not change after acetabular transverse ligament excision (8). Thus, there are many uncertainties regarding the function of the acetabular transverse ligament. However, the acetabular transverse ligament is considered to be a factor that limits femoral head reduction in the developmental dislocation of the hip, and its excision and resection release the acetabular entrance, rendering the femoral head easier to reduce to the acetabulum $(9,10)$. The acetabular transverse ligament is easy to visualize, even in the joints with arthritic changes; thus, it is clinically useful (e.g., as an indicator of acetabular component placement in total hip arthroplasty) (11-13).

Ligaments usually connect to bones; however, the transverse ligament also connects to the anterior and posterior angles of the acetabulum. It is rare for a ligament to appear clearly attached to a tissue other than bone, as noted in the case of the acetabular labrum. Thus, it would be interesting to observe how these two systems are connected. To achieve this goal, the microstructure of the main collagen fiber components of the acetabular transverse ligament must be clarified. Treatment of a deparaffinized block with $2 \mathrm{~N} \mathrm{NaOH}$ enables the digestion of the cellular matrix and observation of the collagen fibers that make up the ligament, cartilage, and bone (14).

By observing these samples three-dimensionally using scanning electron microscopy (SEM), it has become possible to observe the microstructure, namely, the collagen fibers, acetabular labrum, and ligamentum teres of the hip joint $(15,16)$. In addition, type I collagen fibrils in the collagen tissue and tendon have a diameter of approximately 150 and $190 \mathrm{~nm}$, respectively (17). The ligament is predominantly composed of type I collagen $(17,18)$, whereas the diameter of type II collagen fibrils in the hyaline cartilage is $75 \mathrm{~nm}$ on average, and the predominant collagen type is type II $(17,19)$. This knowledge is useful for collagen typing when performing SEM. However, the microstructure of the acetabular transverse ligament itself, namely of its collagen fibers, has not yet been clarified as it has in other ligaments. Furthermore, the mechanism underlying the connection between the histologically distinct collagen fibers of the acetabular transverse ligament and those of the acetabular labrum remains unknown. Therefore, the purpose of this study was to clarify the microstructure of the acetabular transverse ligament in terms of the construction and arrangement of the collagen fibers and their connection to the acetabular labrum using light microscopy (LM) and SEM.

\section{MATERIALS AND METHODS}

The acetabular transverse ligaments and labrums obtained from a 69-year-old woman and an 82-year-old woman during total hip replacement with femoral head necrosis and femoral neck fracture, respectively, were used for the experiments. The acetabular transverse ligaments and labrums were fixed with $10 \%$ formalin immediately after sample collection. After fixation, the specimens were immersed in a 5\% ethylenediaminetetraacetic acid (EDTA; Wako Pure Chemical Industries, Osaka, Japan) solution for 2 weeks for decalcification. The specimens were then cut into 5- to 10 -mm squares, fixed in $10 \%$ formalin or $4 \%$ paraformaldehyde in phosphate buffer ( $\mathrm{pH}$ 7.4), dehydrated, and embedded in paraffin.

\section{LM examination}

Paraffin sections $(5-10 \mu \mathrm{m})$ were stained with hematoxylin and eosin (HE; pH 2.5) or alcian blue (AB; pH 1.0 and 2.5) to assess the carbohydrate reactions histochemically and observed under LM (Nikon ECLIPSE Ci-L, Tokyo, Japan) at $2 \times$ or $4 \times$ magnification.

The slides were deparaffinated and rehydrated with decreasing ethanol passages. Masson's trichrome staining was performed as described below. After washing with running water for 3 minutes and rinsing with distilled water, the slides were treated for 10 minutes with a dedicated mordant (Muto, Tokyo, Japan) for Masson's trichrome staining. Next, the slides were washed with running water for 5 minutes and rinsed with distilled water. To differentiate the nuclei, the slides were then immersed in Carrazzi's hematoxylin for 15 minutes and rinsed in $0.5 \%$ hydrochloric acid water. In order to stain the cytoplasm and erythrocytes, the slides were submerged in Masson B for 15 minutes and then rinsed twice with $1 \%$ acetic acid water. Next, the slides were treated with phosphotungstic acid solution, a mordant, for another 6 minutes. After washing them twice with $1 \%$ acetic acid, the slides were immediately submerged in aniline blue solution for 3 minutes to stain the fibroblasts and collagen. After subsequent washing with $1 \%$ acetic acid twice, the slides were dehydrated quickly in $100 \%$ ethanol, cleared in xylene, and mounted onto coverslips using a mounting medium.

\section{SEM examination}

The remaining paraffin blocks were deparaffinized with xylene overnight and re-fixed with a $2.5 \%$ glutaraldehyde and $2.0 \%$ formaldehyde solution for SEM after observation under an optical microscope. Furthermore, after being immersed in the $2 \mathrm{~N} \mathrm{NaOH}$ solution at $37^{\circ} \mathrm{C}$ for 3 hours to digest the extracellular matrix, the samples were thoroughly washed with physiological saline and placed in $1 \%$ osmium tetroxide, $1 \%$ tannic acid, and $1 \%$ osmium tetroxide for 1 hour each, dehydrated with alcohol, and then freeze-dried with butyl alcohol. The dried specimens were coated with 
a 4-nm thick gold layer (E102 Ion Sputter, Hitachi, Tokyo, Japan) and examined using SEM (S-4800, Hitachi, Tokyo, Japan) at $5 \mathrm{Kv}(14)$.

Fifty collagen fibrils from multiple microphotographs were used to determine the mean diameter of the collagen fibrils using Java ImageJ software version 1.46d (National Institute of Health, Bethesda, MD). The mean chondrocyte size was determined using 25 chondrocytes from different microphotographs at the same magnification $(1000 \times)$. To investigate the acetabular labrum's fibrous cartilage and transverse ligament, the collagen was classified as type I or II based on the diameter of the collagen fibrils (type I, approximately $150 \mathrm{~nm}$; type II, $75 \mathrm{~nm}$ ) (17).

The study procedures were conducted ethically in accordance with international standards, as required by the journal and as described by Padulo et al. (20). This study was approved by the Ethics Committee of Oita University (approval number, 1053; approval date, 22 July 2016). All experimental procedures were performed in accordance with the Declaration of Helsinki. Informed consent was obtained from all patients for study participation and publication of their clinical information.

\section{RESULTS}

\section{Macroscopic findings}

A schematic drawing of the constitution of the acetabular labrum and transverse ligament is shown in figure 1a. In a normal joint, tissues of the acetabular transverse ligament and the labrum are continuous with one another (figure $\mathbf{1 b}$ ).

\section{LM findings}

The acetabular transverse ligament is composed of bundles of collagen fibers and was, thus, eosinophilic in HE-stained specimens but negatively stained in AB-stained $(\mathrm{pH} 1.0$ and 2.5) specimens. In contrast, the acetabular labrum is composed of fibrocartilage; thus, it stained bright in HE-stained specimens and strongly in AB-stained specimens (figure 2). The collagen fibers of the acetabular transverse ligament appeared to be penetrating and surrounding the cartilage tissue of the acetabular labrum in enlarged LM images of the connection of both tissues (figure 3). Masson trichrome staining revealed clear wave-depressing collagen fibers in the acetabular transverse ligament (figure 4).

\section{SEM examination}

In low-magnification SEM images of the acetabular transverse ligament, bundles of 30-50- $\mu \mathrm{m}$ wide collagen fibers were arranged in parallel (figure 5). The collagen fibrils composing the acetabular transverse ligament had a mean diameter of $161.28 \pm 16.79$ (range: $133.3-189.7$ ) nm, forming collagen fibrils classified as type I (figure 6).

In SEM images of the acetabular labrum, the cartilage tissue was composed of chondrocytes and extracellular matrix (figure 7). In high-magnification SEM images of the chon- a

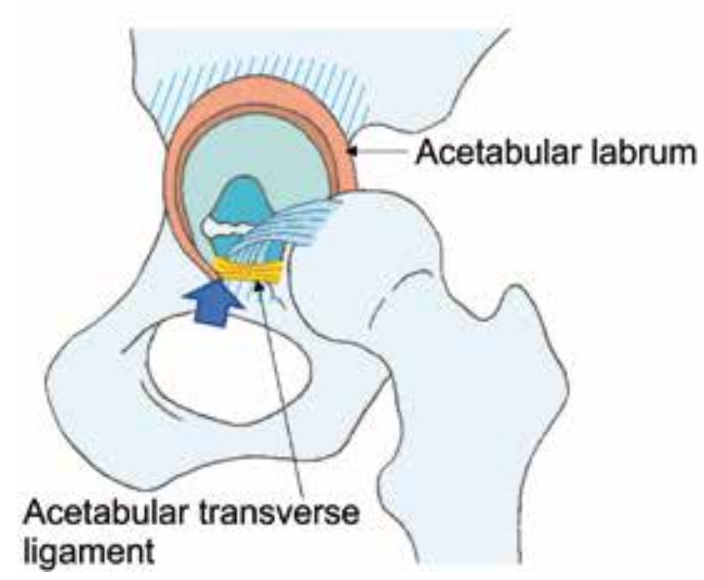

b

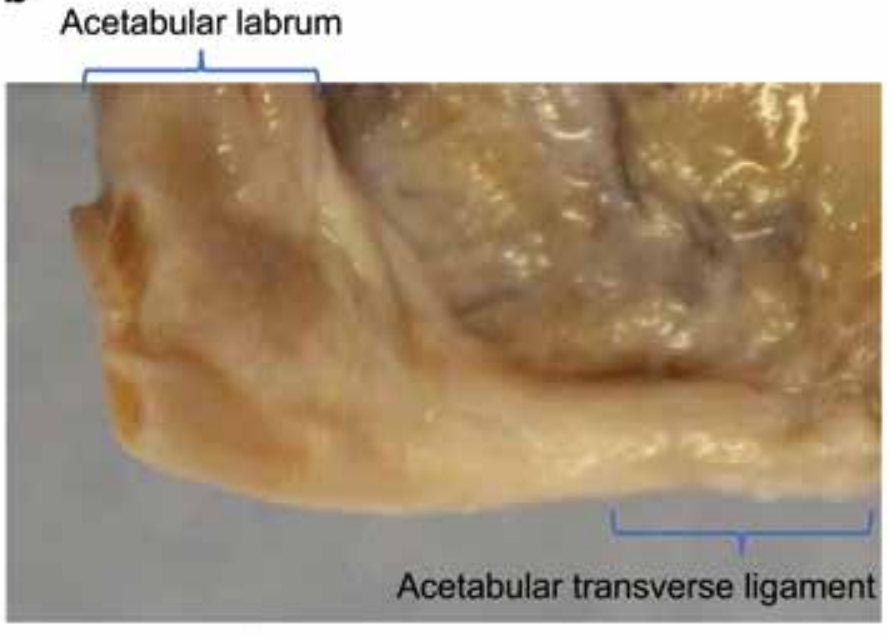

Figure 1. Macroscopic appearance of the transitional part of the acetabular transverse ligament $(\mathrm{L})$ and acetabular labrum (AL). a. The large arrow in the schematic drawing indicates the junction between the $A L$ and the $L$.

b. Both tissues appear to be connected without clear boundaries. 

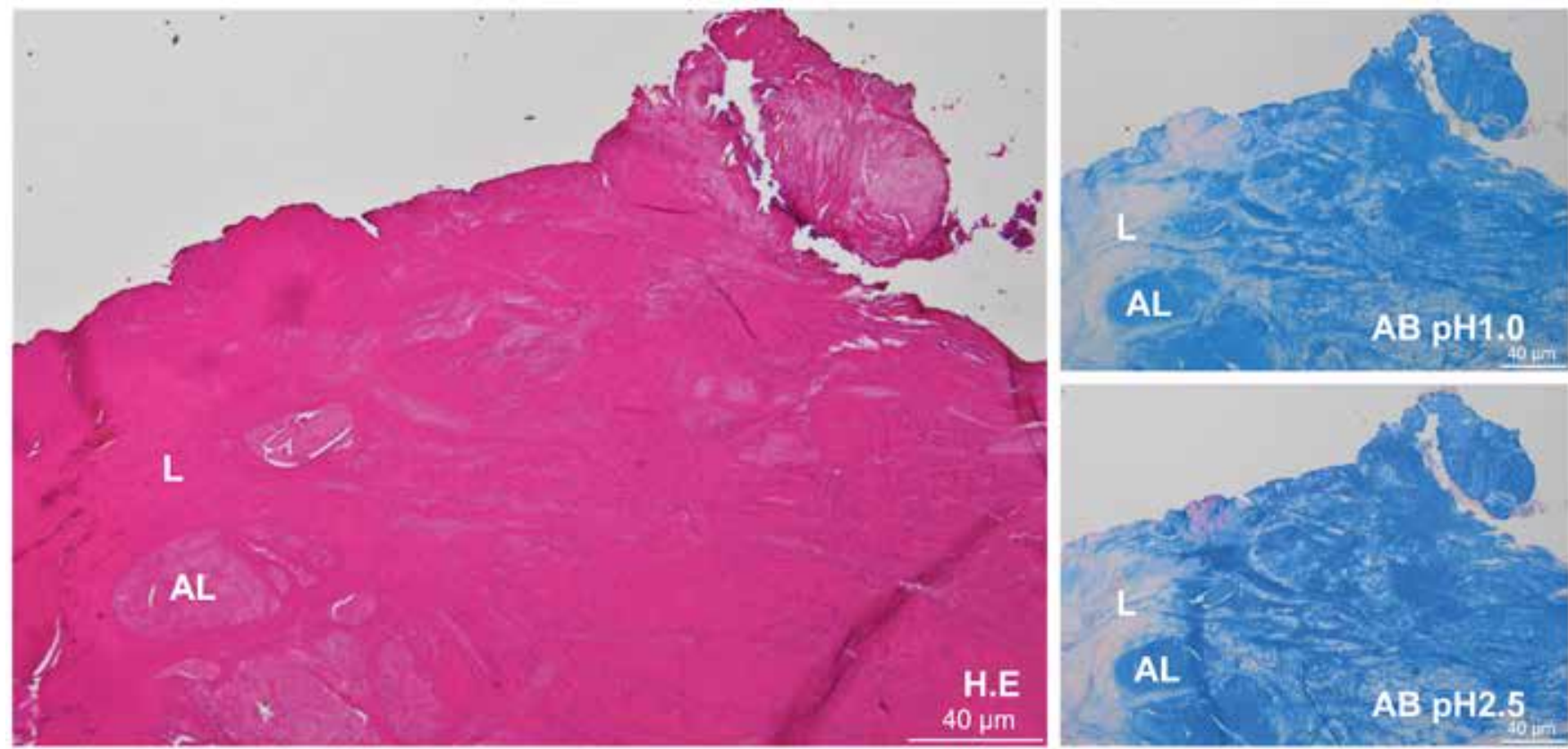

Figure 2. Optical microscopy images of the transition between the acetabular transverse ligament and labrum. The acetabular transverse ligament $(\mathrm{L})$ consists of collagen fibers and stains negative on alcian blue (AB) staining (pH 1.0 and 2.5). The acetabular labrum $(\mathrm{AL})$, on the other hand, is composed of fibrocartilage, stains slightly brighter on eosin staining, and is strongly positive for $A B$ staining (pH 1.0 and 2.5).
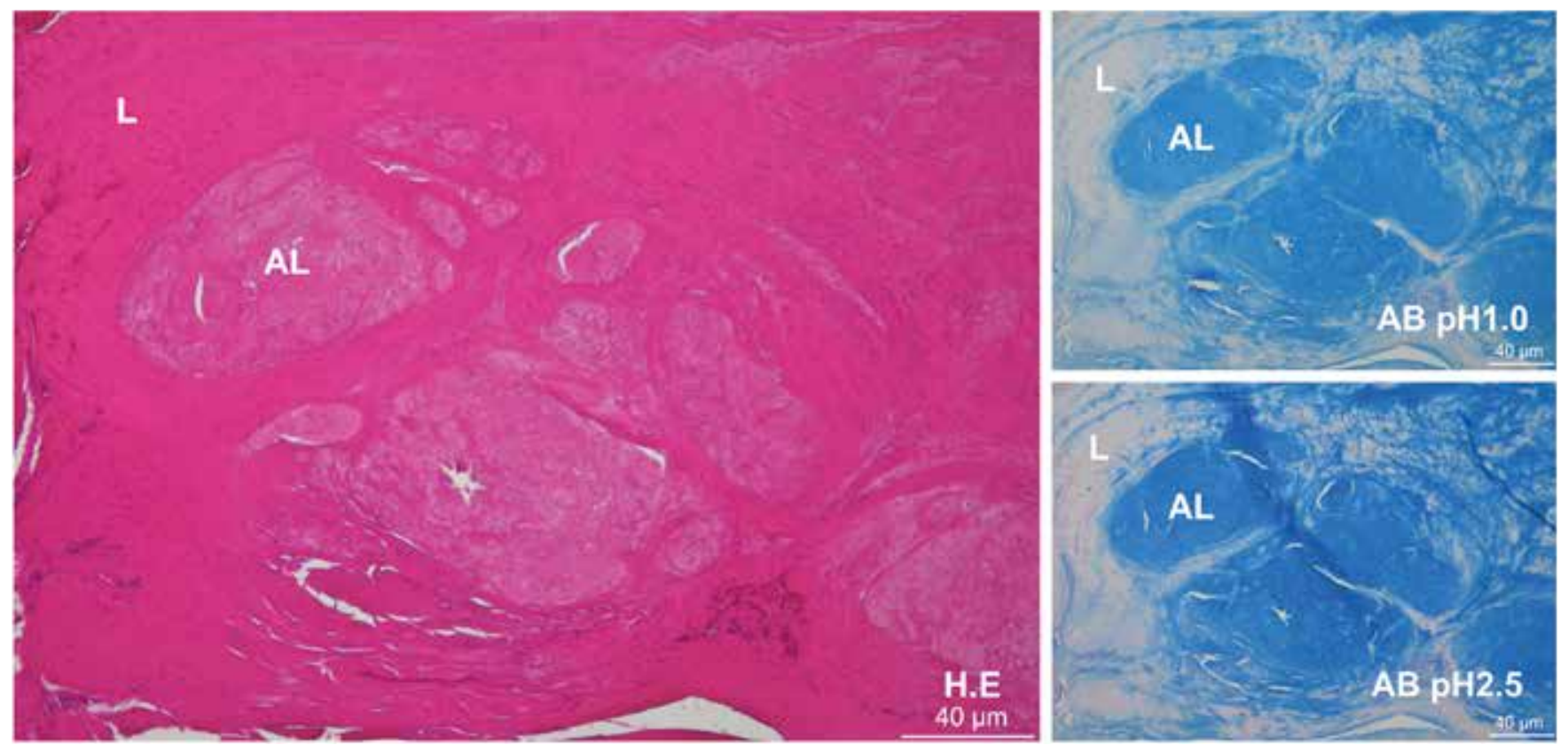

Figure 3. Magnified optical microscopy images of the transitional part of the acetabular transverse ligament $(\mathrm{L})$ and acetabular labrum (AL). Collagen fibers composing the $\mathrm{L}$ appear to penetrate the cartilage tissue. 


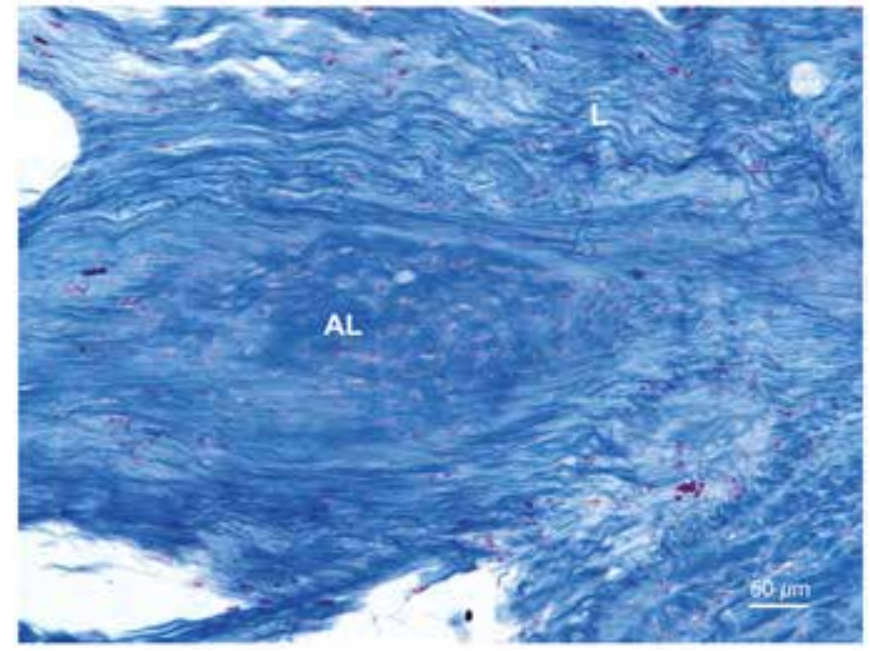

Figure 4. Optical microscopy image of the transition between the acetabular transverse ligament $(L)$ and acetabular labrum (AL) with Masson's trichrome staining. The $L$ shows clear wave-depressing fibers, while the $\mathrm{AL}$ shows rich cellular components around; no clear wave-depressing fibers appear to be mixed at the junction of the two tissue types.

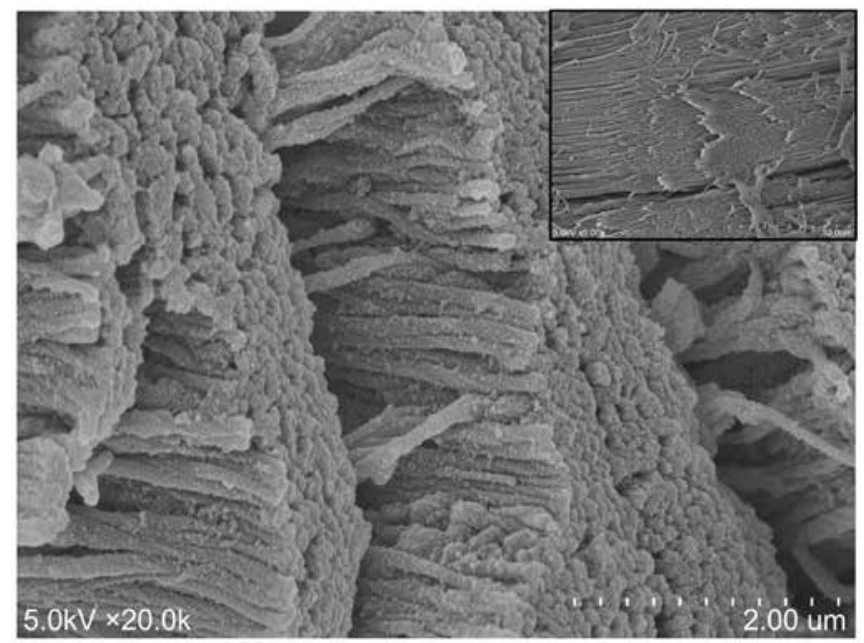

Figure 6. Horizontal ligament collagen fibers in high-magnification scanning electron microscopy (SEM) images. As treatment with $2 \mathrm{~N} \mathrm{NaOH}$ digests the extracellular matrix, the collagen fibrils that make up the collagen fibers are clearly observed in the inserted figure. The type I collagen fibrils are arranged in parallel and have a mean diameter of 161.28 \pm 16.79 (range: 133.3-189.7) nm.

drocytes and extracellular matrix, the extracellular matrix was densely packed with type II collagen, with a chondrocyte size of 12.52 \pm 1.71 (range: 10-15.17) $\mu \mathrm{m}$ (figure 8).

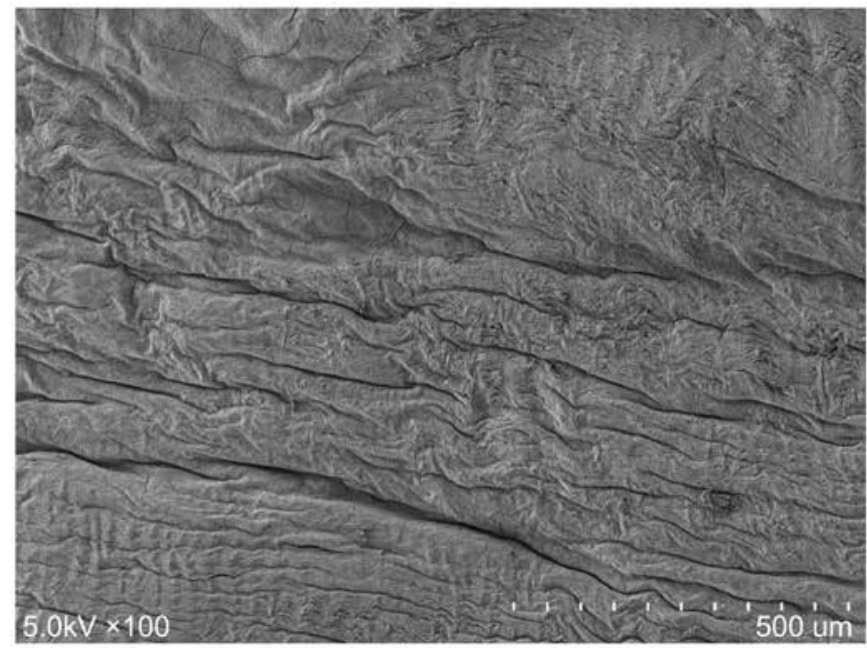

Figure 5. Low-magnification scanning electron (SEM) microscopy image of the acetabular transverse ligament $(L)$. The $L$ consists of bundles of collagen fibers with a width of 30-50 $\mathrm{nm}$ that are arranged in parallel.

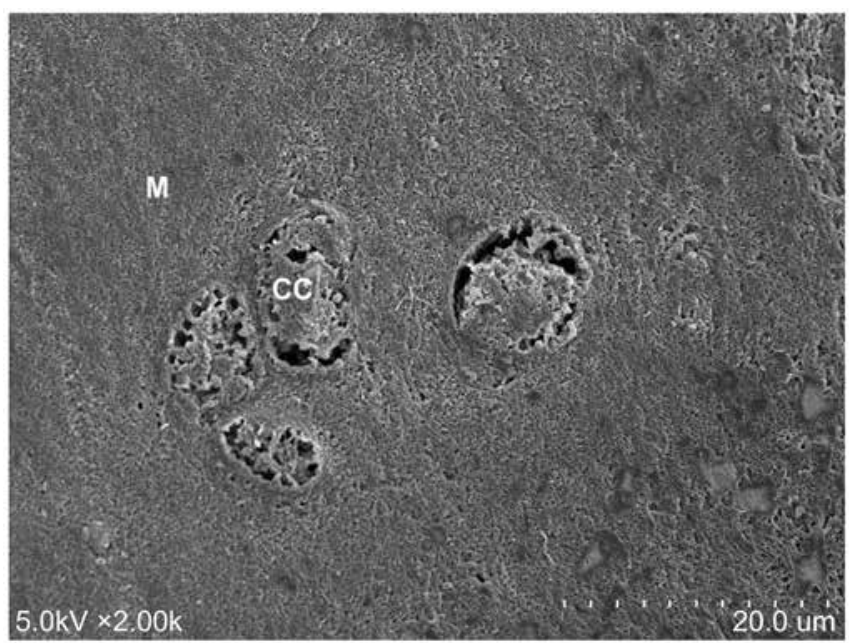

Figure 7. Scanning electron microscopy (SEM) image of the joint lip cartilage tissue. The cartilage tissue consists of chondrocytes (CC) and extracellular matrix (M).

Type I collagen was also present in the acetabular labrum and appeared to cause cartilage tissue disruption, showing a parallel arrangement as seen in the transverse ligament. 


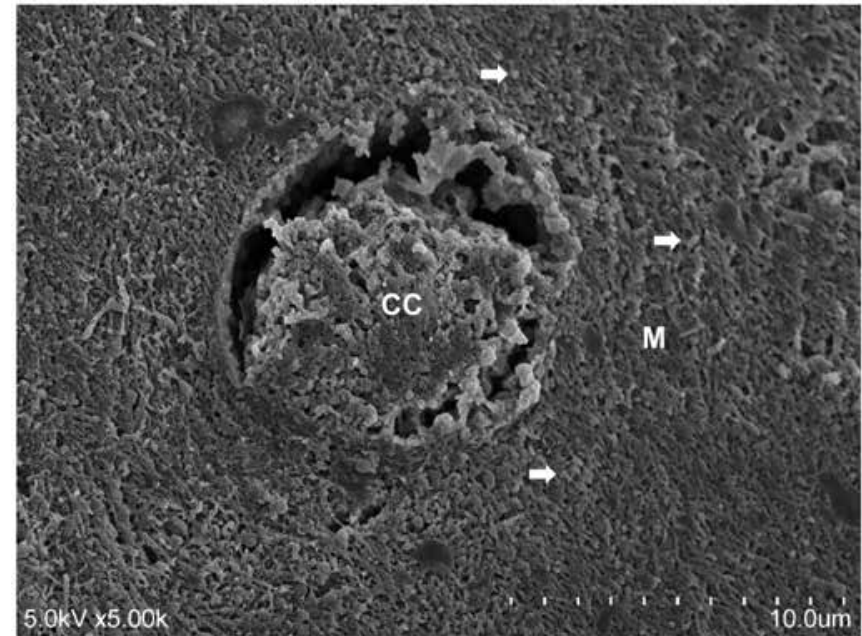

Figure 8. High-magnification scanning electron microscopy (SEM) image of the chondrocytes (CC) and extracellular matrix (M). In the $M$, the thinner collagen fibrils are dense and have a major axis of $12.52 \pm 1.71$ (range: 10-15.17) $\mu \mathrm{m}$.

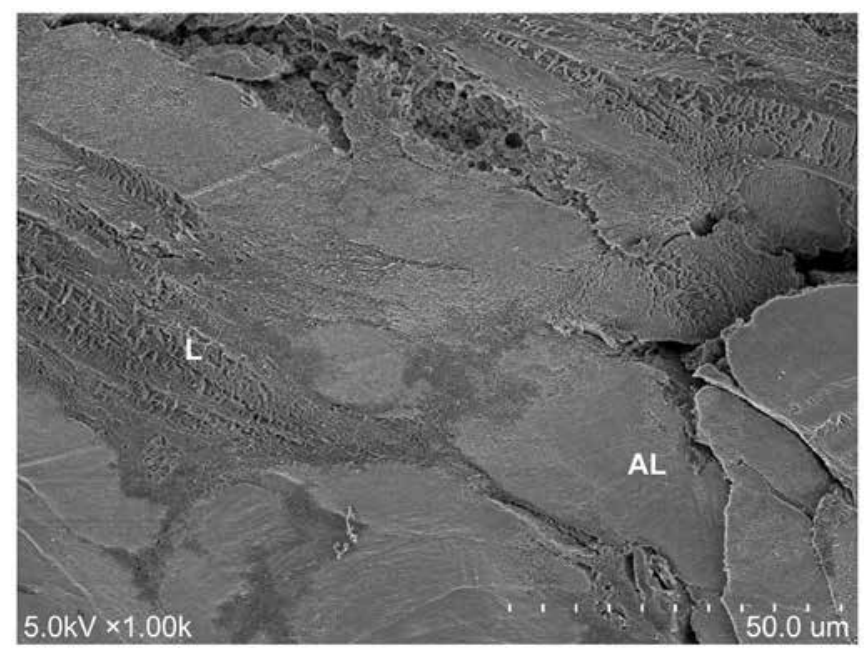

Figure 10. Scanning electron microscopy (SEM) image of the junction between the acetabular transverse ligament $(L)$ and the acetabular labrum (AL). The $L$ is penetrated by cartilage tissue that constitutes the AL.

Ultra-low magnification SEM images of the transitional zone between the acetabular transverse ligament and acetabular labrum revealed that the acetabular transverse ligament penetrated the cartilage tissue composing the acetabular labrum, as seen in LM images (figure 9). Furthermore, the collagen fibers of the acetabular trans-

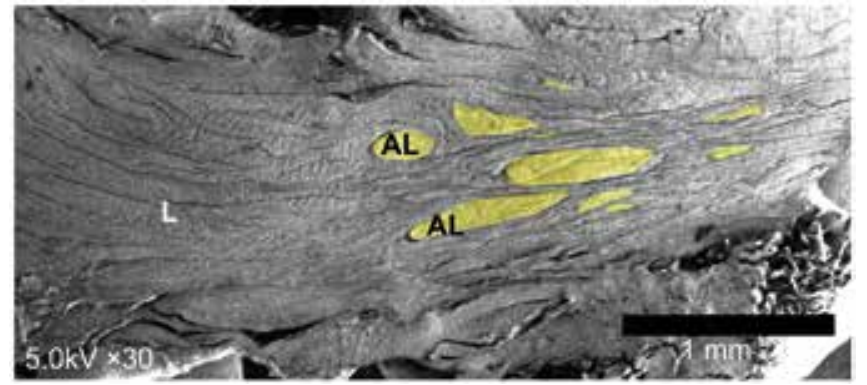

Figure 9. Ultra-low magnification scanning electron microscopy (SEM) image of the transition zone between the acetabular transverse ligament $(L)$ and acetabular labrum $(A L)$. The cartilage tissues are colored to differentiate between both tissues in the $L$ and $A L$. The tissues of the $A L$ seem to penetrate the tissues of the $\mathrm{L}$.

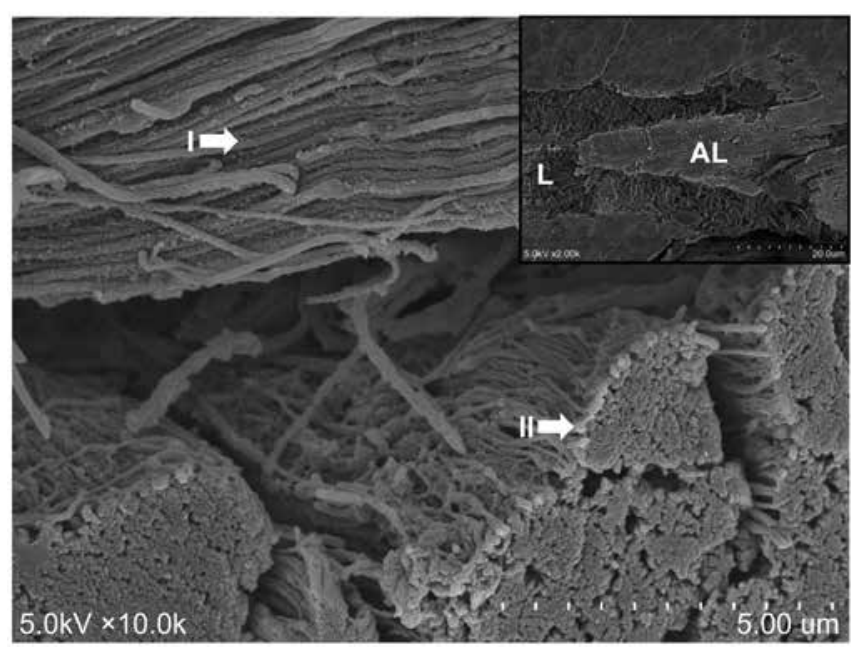

Figure 11. Scanning electron microscopy (SEM) images of the collagen fibrils in contact with the cartilage tissue. The collagen fibrils that constitute the acetabular transverse ligament $(L)$ are dense and surround the cartilage tissue that constitutes the acetabular labrum (AL) in the inserted figure. Higher magnification SEM images show that type II collagen of the $A L$, with a diameter of 78.08 \pm 13.04 (range: 61.8-100.5) $\mathrm{nm}$, did not directly bind to type I collagen constituting the $\mathrm{L}$.

verse ligament were interspersed in the acetabular labrum cartilage (figure 10); they also surrounded and were densely surrounded by cartilage (figure 11). At the junction of the acetabular transverse ligament and acetabular labrum, type I collagen of the transverse ligament and type II collagen of the acetabular labrum, which had a diameter of 
$73.65 \pm 8.56$ (range: $61.8-89.56) \mathrm{nm}$, did not bind directly. Instead, the collagen fibers of the acetabular transverse ligament and labrum were interwoven.

\section{DISCUSSION}

We assessed the microstructure of the acetabular transverse ligament with respect to the construction and arrangement of the collagen fibers constituting the ligament and their connection with the acetabular labrum. We found that the collagen fibers were interwoven between the cartilage tissues of the acetabular labrum; these structures seemed to be directly joined histologically with one another. The SEM images, however, revealed that the collagen fibers were not directly connected; instead, the fine collagen fibers with varying diameters extending from the structures were interwoven.

The present study revealed the microstructure of the acetabular transverse ligament and its connection with the acetabular labrum by delineating collagen for the first time using SEM. A limitation of this study is that the effects of sex and age were not evaluated because only two samples were collected, and both subjects were older women. We also did not evaluate the attachment between the acetabular transverse ligament and bone, which may have indicated a different histological connection from that of the present result.

The acetabular labrum has abundant sensory fibers, mechanoreceptors, and blood vessels in the fibrous connective tissue (16). Kapetanakis et al. also found that free nerve endings and nerve end organ presence was greater in the ventral side of the acetabular labrum (21). On the other hand, although Gerhardt et al. reported that sensory fibers and mechanoreceptors are present in the acetabular transverse ligament using LM after histological staining (22), Kiliçarslan et al. concluded that it showed good vascularity with abundant free nerve fibers within the fibrous connective tissue; however, the latter group of authors did not find mechanoreceptors using neurofilament protein and S-100 protein immunohistochemistry and microscopy (23). In addition, no chondrocytes have been reported to be present in the transverse ligament, unlike in the acetabular labrum (24). However, very little is known about the microstructure of the acetabular transverse ligament. The acetabular transverse ligament is unusual in that it is a ligament that connects to tissues other than bone.

In the present study, the type I collagen fibrils were found to form bundles and collagen fibers, had a width of 30-50 $\mu \mathrm{m}$, and were arranged in parallel in the acetabular transverse ligament, as seen in other ligaments. The present study also revealed that the histological features of the acetabular transverse ligament were completely different from those of the cartilage tissue of the acetabular labrum, which is mainly composed of type II collagen fibers. The diameter of the type I fibrils of the acetabular transverse ligament was, on average, $161.28 \pm 16.79$ (range: $133.3-189.7$ ) $\mathrm{nm}$ in this study, which is greater than that of general type I collagen and lower than that of tendon collagen (12). In contrast, although the diameter of the type II fibrils in the acetabular labrum, which have a fibrous cartilage structure, was $73.65 \pm 8.56$ (range: $61.8-89.56) \mathrm{nm}$, it was similar to that of the collagen fibrils in the hyaline cartilage(17). Further, the collagen fibers of the acetabular transverse ligament were interwoven with the acetabular labrum cartilage, forming a layered structure with collagen fibers of different diameters. These components histologically seemed to be directly joined to each other. However, in high-magnification SEM images, the diameter of the collagen fibers was rather uniform, and their distribution patterns were well segregated from the bundles composed of smaller fibers. Nogami et al. (16) reported that multiple layers of thin type I collagen are interwoven with the cartilage tissue of the acetabular labrum. Conversely, in the adhesive portion of the acetabular transverse ligament, many relatively thick bundles consisting of type I collagen were present and formed a layered structure. Therefore, type II and type I collagen can coexist and seem to give rise to various structures with distinct histological features. Regarding the attachment of the ligament to tissues other than bone, it has been found that a part of the ligamentum teres composed of type I collagen is attached to the surface and inside of the articular cartilage of the femoral head (15).

\section{CONCLUSIONS}

The present study revealed the microstructure of the transverse ligament and its attachment to the acetabular labrum. In the macrostructure of the acetabular transverse ligament, collagen fibers with a width of 30-50 $\mu \mathrm{m}$ were arranged in parallel which consisted of bundle with type I collagen fibrils. The acetabular transverse ligament had a distinctly different microstructure than the acetabular labrum with cartilage tissues. In the connection between the acetabular transverse ligament and the labrum, it was found that the microstructure was not a direct adhesion, but rather a structural bond, at least with respect to collagen. Although ligaments usually attach to bone, the results suggest that ligaments can attach to tissues other than bone. As this study only reports findings based on morphological observation, quantitative analysis, such as determining the number of cells or conducting protein analysis, should be performed in detail in future studies. New findings from future studies will contribute to the elucidation of the mechanism underlying the connection of different tissues. 


\section{UNBLINDED ETHICS STATEMENT}

The study procedures were conducted ethically in accordance with international standards, as required by the journal and as described in Padulo et al. 15. This study was approved by the Ethics Committee of Oita University (approval number, 1053; approval date, 22 July 2016). All experimental procedures were performed in accordance with the Declaration of Helsinki. Informed consent was obtained from all patients for study participation and publication of their clinical information.

\section{ACKNOWLEDGEMENTS}

We would like to thank Editage (www.editage.com) for English language editing.

\section{CONFLICT OF INTERESTS}

The authors declare that they have no conflicts of interests.

\section{REFERENCES}

1. Löhe F, Eckstein F, Sauer T, Putz R. Structure, strain and function of the transverse acetabular ligament. Acta Anat (Basel) 1996;157:315-323.

2. Aprato A, Giachino M, Masse A. Arthroscopic approach and anatomy of the hip. Muscles Ligaments Tendons J 2016;6:309. 316.

3. Bolia I, Chahla J, Locks R, Briggs K, Philippon MJ. Microinstability of the hip: a previously unrecognized pathology. Muscles Ligaments Tendons J 2016;6:354-360.

4. Tran Trung D, Pham Trung H, Truong Cong M, Maffulli N, Nguyen Trung T. The anatomical characteristics of Vietnamese adult hip joint: a multiplanar reconstruction computer tomographic study. Muscles Ligaments Tendons J 2019;9:165-172.

5. Chmielewski J, Albiñana J. Failures of open reduction in developmental dislocation of the hip. J Pediatr Orthop B 2002;11:284-289.

6. Gala L, Clohisy JC, Beaulé PE. Hip Dysplasia in the Young Adult. J Bone Joint Surg Am 2016;98:63-73.

7. Bullough P, Goodfellow J, Greenwald AS, O'Connor J. Incongruent surfaces in the human hip joint. Nature 1968;217:1290.

8. Konrath GA, Hamel AJ, Olson SA, Bay B, Sharkey NA. The role of the acetabular labrum and the transverse acetabular ligament in load transmission in the hip. J Bone Joint Surg Am 1998;80:1781-1788.

9. McCluskey WP, Bassett GS, Mora-Garcia G, MacEwen GD. Treatment of failed open reduction for congenital dislocation of the hip. J Pediatr Orthop 1989;9:633-639.
10. Hsieh SM, Huang SC. Treatment of developmental dysplasia of the hip after failed open reduction. J Formos Med Assoc 1998;97:763-769.

11. Archbold HA, Mockford B, Molloy D, McConway J, Ogonda L, Beverland D. The transverse acetabular ligament: an aid to orientation of the acetabular component during primary total hip replacement: a preliminary study of 1000 cases investigating postoperative stability. J Bone Joint Surg Br 2006;88:883 886.

12. Fujita K, Kabata T, Maeda T, et al. The use of the transverse acetabular ligament in total hip replacement: An analysis of the orientation of the trial acetabular component using a navigation system. Bone Joint J 2014;96-B:306-311.

13. Griffin AR, Perriman DM, Bolton CJ, Smith PN. An in vivo comparison of the orientation of the transverse acetabular ligament and the acetabulum. J Arthroplasty 2014;29:574-579.

14. Shimada T, Sato F, Zhang L, Ina K, Kitamura H. Three-dimensional visualization of the aorta and elastic cartilage after removal of extracellular ground substance with a modified $\mathrm{NaOH}$ maceration method. J Electron Microsc 1993;42:328333.

15. Kaku N, Shimada T, Tabata T, et al. Three-dimensional architecture of the ligamentum teres in the human hip joint. Muscles Ligaments Tendons J 2018;7:442-448.

16. Nogami R, Kaku N, Shimada T, Tabata T, Tagomori H, Tsumu$\mathrm{ra} \mathrm{H}$. Three-dimensional architecture of the acetabular labrum in the human hip joint. Med Mol Morphol 2019. doi:10.1007/ s00795-019-00228-3.

17. Fawcett DW, Bloom W. A textbook of histology. New York/ London; Chapman \& Hall. 1994.

18. Kew SJ, Gwynne JH, Enea D, et al. Regeneration and repair of tendon and ligament tissue using collagen fibre biomaterials. Acta Biomater 2011;7:3237-3247.

19. Almarza AJ, Athanasiou KA. Design characteristics for the tissue engineering of cartilaginous tissues. Ann Biomed Eng 2004;32:2-17.

20. Padulo J, Oliva F, Frizziero A, Maffulli N. Muscles, Ligaments and Tendons Journal - Basic principles and recommendations in clinical and field Science Research: 2016 Update. Muscles Ligaments Tendons J 2016;19:1-5.

21. Kapetanakis S, Gkantsinikoudis N, Dermon A, Kommata V, Papathanasiou J, Soukakos P, Dermon C. Normal microscopic architecture of acetabular labrum of hip joint: a qualitative original study with clinical aspects. Muscles Ligaments Tendons J 2017;7:279-285.

22. Gerhardt M, Johnson K, Atkinson R, et al. Characterisation and classification of the neural anatomy in the human hip joint. Hip Int 2012;22:75-81.

23. Kılıçarslan K, Kılıçarslan A, Demirkale İ, Aytekin MN, Aksekili MA, Uğurlu M. Immunohistochemical analysis of mechanoreceptors in transverse acetabular ligament and labrum: a prospective analysis of 35 cases. Acta Orthop Traumatol Turc 2015;49:394-398.

24. Gray H, Lewis WH. Anatomy of the Human Body. Philadelphia; Lea \& Febiger 2010 